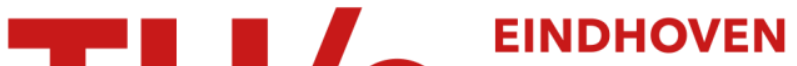 UNIVERSITY OF TECHNOLOGY
}

\section{Coupled singlet-triplet analysis of two-color cold-atom photoassociation spectra}

Citation for published version (APA):

Vogels, J. M., Freeland, R. S., Tsai, C. C., Verhaar, B. J., \& Heinzen, D. J. (2000). Coupled singlet-triplet analysis of two-color cold-atom photoassociation spectra. Physical Review A : Atomic, Molecular and Optical Physics, 61(4), 043407-1/11. [043407]. https://doi.org/10.1103/PhysRevA.61.043407

DOI:

10.1103/PhysRevA.61.043407

Document status and date:

Published: 01/01/2000

\section{Document Version:}

Publisher's PDF, also known as Version of Record (includes final page, issue and volume numbers)

\section{Please check the document version of this publication:}

- A submitted manuscript is the version of the article upon submission and before peer-review. There can be important differences between the submitted version and the official published version of record. People interested in the research are advised to contact the author for the final version of the publication, or visit the $\mathrm{DOI}$ to the publisher's website.

- The final author version and the galley proof are versions of the publication after peer review.

- The final published version features the final layout of the paper including the volume, issue and page numbers.

Link to publication

\section{General rights}

Copyright and moral rights for the publications made accessible in the public portal are retained by the authors and/or other copyright owners and it is a condition of accessing publications that users recognise and abide by the legal requirements associated with these rights.

- Users may download and print one copy of any publication from the public portal for the purpose of private study or research.

- You may not further distribute the material or use it for any profit-making activity or commercial gain

- You may freely distribute the URL identifying the publication in the public portal.

If the publication is distributed under the terms of Article 25fa of the Dutch Copyright Act, indicated by the "Taverne" license above, please follow below link for the End User Agreement:

www.tue.nl/taverne

Take down policy

If you believe that this document breaches copyright please contact us at:

openaccess@tue.nl

providing details and we will investigate your claim. 


\title{
Coupled singlet-triplet analysis of two-color cold-atom photoassociation spectra
}

\author{
J. M. Vogels, ${ }^{1}$ R. S. Freeland, ${ }^{2}$ C. C. Tsai, ${ }^{2}, *$ B. J. Verhaar, ${ }^{1}$ and D. J. Heinzen ${ }^{2}$ \\ ${ }^{1}$ Eindhoven University of Technology, Box 513, 5600 MB Eindhoven, The Netherlands \\ ${ }^{2}$ Department of Physics, The University of Texas, Austin, Texas 78712
}

(Received 16 December 1998; published 8 March 2000)

\begin{abstract}
We describe a method that is well suited to analysis of the bound states of the alkali-metal dimers near their dissociation limit. The method combines inverse perturbation theory, coupled-channel bound-state theory, and the accumulated phase method to treat the short-range part of the molecular potentials. We apply this method to analyze the bound-state energies measured in a two-color photoassociation experiment in an ultracold ${ }^{85} \mathrm{Rb}$ gas. This analysis yields information on the interactions between ultracold ${ }^{85} \mathrm{Rb}$ atoms that is important to the understanding of ultracold $\mathrm{Rb}$ collisions and Bose-Einstein condensation.
\end{abstract}

PACS number(s): 32.80.Pj, 34.50.Rk, 33.70.Ca

\section{INTRODUCTION}

The techniques of laser cooling and evaporative cooling have opened the field of ultracold-atom physics for alkalimetal atoms, with spectacular research subjects such as Bose-Einstein condensation, laser-cooled atomic clocks, and atom lasers. It is generally realized that atom interaction processes play a key role in many of these experiments. It is therefore important to obtain a complete, consistent picture of these interactions. In this paper we describe and apply a theoretical method to obtain information on the interactions between cold atoms, making use of measured energies of bound diatomic states. The method has much in common with inverse perturbation analysis (IPA) [1], previously used to obtain a single adiabatic Born-Oppenheimer interaction potential curve from bound-state energies of that potential. In contrast to the IPA it is applicable to cases where different electronic states are strongly coupled, so that the BornOppenheimer approximation breaks down. Although the formalism can be considered as a generalized, coupled version of the IPA, we apply it only in a restricted form: the extraction of information on collisions of ultracold atoms from the bound-state spectrum close to the dissociation threshold. In particular, we apply our method to the highest bound states of ${ }^{85} \mathrm{Rb}_{2}$, which have recently been measured with two-color photoassociation spectroscopy [2]. In this case the molecular singlet and triplet states are strongly coupled by the hyperfine interaction. A brief report of this work has been given previously [2]; in this paper we give a more extensive description of the experimental and theoretical method and of the results.

In the case of ground-state alkali-metal atoms there are two short-range adiabatic Born-Oppenheimer potentials, corresponding to total electronic spin $S=0$ (singlet potential) or $S=1$ (triplet potential). Along the lines of our previous work, at small interatomic distances we do not describe the singlet and triplet potentials in detail, but summarize that information in the form of the phases accumulated $[3,4]$ by the associated rapidly oscillating $S=0$ and $S=1$ radial wave

\footnotetext{
*Present address: National Chung-Kung University, Tai-Nan, Taiwan.
}

functions up to a radius $r_{0}$, which is chosen between $16 a_{0}$ and $20 a_{0}$ depending on the alkali-metal atom considered. It is well known that both cold collision properties and the precise positions of the highest molecular bound states are extremely sensitive to very small changes of the $r<r_{0}$ potentials. Except for the lighest alkali-metal species, potentials in this range are not known with sufficient accuracy to allow for reliable application to cold collisions. Fortunately, it is possible to avoid this difficulty by using boundary conditions at $r_{0}$ in the form of accumulated singlet and triplet phases that summarize the short-range collisional information. In our approach we search for optimal values of the interaction parameters in the range $r>r_{0}$ and of the accumulated phases.

The main feature of our approach is that it can cope with bound states near dissociation, which often show strong singlet-triplet mixing by the hyperfine interaction. Clearly, the higher mixed states can be expected to bear a greater resemblance to the nearby states in the continuum and can therefore more profitably be used for obtaining information on cold collisions by extrapolation through the dissociation threshold. We also emphasize that we carry out a simultaneous analysis for the singlet and triplet parts of spin space. This is important in view of the fact that the singlet and triplet potentials have certain parameters in common, such as the position of the dissociation threshold and the dispersion coefficients. A separate analysis may therefore lead to inconsistencies, such as a crossing of the singlet and triplet potentials at long range [5]. A further feature of our method, connected with its restricted application to the highest bound states, is that the potential variations searched for extend over longer radial intervals. This applies both to the interactions in the range $r<r_{0}$, since they are effectively described via accumulated phases, and to the $r>r_{0}$ interactions, which are described by analytic expressions for dispersion and exchange contributions. In this way we do not run into the pitfalls associated with applications of the IPA to the highest bound levels where nonphysical fluctuations in the local potentials over short distances, of the order of the distance between outer turning points of successive ro-vibrational levels, are difficult to avoid in the search for improved potentials.

One-color cold-atom photoassociation experiments have yielded a great deal of information on the interactions be- 


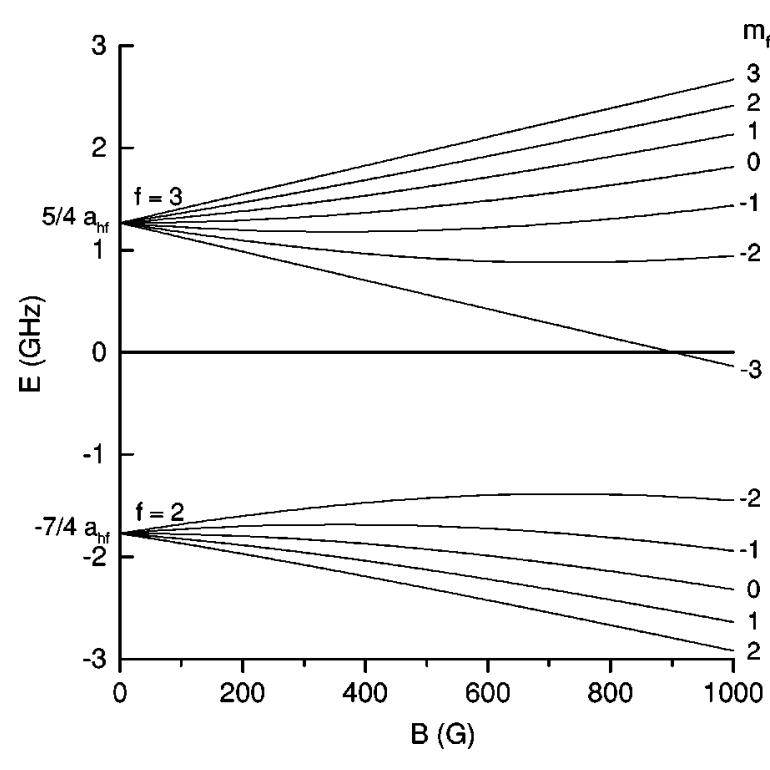

FIG. 1. Breit-Rabi diagram of ${ }^{85} \mathrm{Rb}$ atomic ground state.

tween alkali-metal atoms [6,7]. In these experiments, a tunable laser excites transitions between the initially free state of a pair of colliding, laser-cooled ground-state atoms and excited bound molecular levels of those atoms. These experiments directly yield the level structure of the electronically excited states. The line shapes and strengths of one-color photoassociation spectra have also provided information on the properties of the collisional ground state for ultracold $\mathrm{Rb}$ [8-12], $\mathrm{Na}$ [13], and $\mathrm{Li}$ [14]. More direct information on ground-state cold collision properties can be obtained through direct measurement and analysis of the highest bound levels of the electronic ground state. This can be accomplished through two-color photoassociation spectroscopy, as shown in Fig. 1 of Ref. [2]. A laser at a fixed frequency $\nu_{1}$ excites transitions from the collisional ground state to a particular excited level. A second tunable laser of frequency $\nu_{2}$ couples this excited level back to the vibrational levels of the electronic ground state. This yields a spectrum of these high-lying bound levels. Experiments of this kind have been completed for $\mathrm{Rb}$ [2], $\mathrm{Na}$ [15], and $\mathrm{Li}$ [16].

In our $\mathrm{Rb}$ two-color photoassociation experiments, we select a specific initial two-atom spin state by carrying out the experiment on a doubly polarized gas sample, i.e., with maximum projections of electronic and nuclear spins of an atom along a quantization direction $z$. The atoms occupy the highest hyperfine state of the ${ }^{85} \mathrm{Rb}$ atomic Breit-Rabi diagram (see Fig. 1). As the excited electronic state we select the $0_{g}^{-}$state asymptotically connected to the $5^{2} S_{1 / 2}$ $+5^{2} P_{1 / 2}^{g}$ dissociation limit [17]. (We use the notation $\Omega_{\sigma}^{ \pm}$, where $\Omega$ is the absolute value of the projection of the total electronic angular momentum along the molecular axis, $\sigma$ $=g$ or $u$ is the electronic parity, and \pm denotes the reflection symmetry for $\Omega=0$.) In this way we avoid a complex hyperfine "spaghetti" of excited states [18], since the nuclear spins in this $\Omega=0$ state are decoupled to a very good approximation from the remaining molecular degrees of freedom. Moreover, it turns out that the $0_{g}^{-}\left(S+P_{1 / 2}\right)$ electronic state has the advantage that the two $1 / 2$ angular momenta of the $S_{1 / 2}$ and $P_{1 / 2}$ atomic states are coupled to give a vanishing two-atom electronic angular momentum $j=0$. The total molecular angular momentum $\vec{J}$ in the excited state (excluding the nuclear spins) thus equals the orbital angular momentum $\vec{l}$ of the collision. The resulting $J=l$ selection rule has been of considerable help in the past to simplify the analysis of our previous one-color photoassociation experiments [8-10]. We note that this is not a general property of $\Omega$ $=0$ states. For example, for the $0_{g}^{-}$"pure long-range state" connecting to the $5{ }^{2} S_{1 / 2}+5^{2} P_{3 / 2}$ limit [17,19], the atomic angular momenta $1 / 2$ and $3 / 2$ do not couple to $j=0$. Even for the lower $0_{g}^{-}$state the $J=l$ selection rule is valid only for levels with binding energies small compared to the $P_{1 / 2}$ $-P_{3 / 2}$ fine-structure splitting $\left(238 \mathrm{~cm}^{-1}\right)$, since otherwise the $S+P_{1 / 2}$ and $S+P_{3 / 2}$ electronic states are too strongly mixed by the resonant electric dipole interaction. The excited levels that are involved in the photoassociation process studied in this paper fulfill this requirement. Selecting the $0_{g}^{-}(S$ $\left.+P_{1 / 2}\right)$ state, we define not only the partial wave channel $l$ $=J$ from which the excitation occurs, but also the rotational $l$ value of the final $\mathrm{Rb}_{2}$ bound states formed.

Even with this simplification the observed bound-state spectrum is expected to be rather complex. In particular, the complexity arises from the fact that the total spins $f_{1}$ and $f_{2}$ $(=2$ or 3 , see Fig. 1) of the separated atoms are not conserved during a collision. These quantum numbers are only good at long range. At smaller distances the exchange interaction mixes the $\left(f_{1}, f_{2}\right)$ quantum numbers. At short range where it dominates, the molecular quantum numbers $(S, I)$, with $\vec{S}=\vec{s}_{1}+\vec{s}_{2}$ the total electron spin and $\vec{I}=\vec{i}_{1}+\vec{i}_{2}$ the total nuclear spin, are good quantum numbers. For not too strong $B$ fields the total angular momentum $\vec{F}=\vec{f}_{1}+\vec{f}_{2}$ is conserved at all distances. Figure 2 of Ref. [2] shows adiabatic molecular potentials for ${ }^{85} \mathrm{Rb}_{2}$ with the pure triplet $F=6$ potential subtracted off. The change between molecular and atomic (hyperfine) coupling occurs between $19 a_{0}$ and $26 a_{0}$ for the ${ }^{85} \mathrm{Rb}+{ }^{85} \mathrm{Rb}$ system. The above-mentioned radius $r_{0}$ is chosen at the left-hand boundary of this range, because we want $S$ to be a good quantum number up to this point. Starting from $r_{0}$, the radial motion on the potentials is not perfectly adiabatic, so that curves with the same $F$ must be treated as a coupled-channel problem. Often, an $r$-independent diabatic basis of pure $(S, I)$ or pure $\left(f_{1}, f_{2}\right)$ states is the most convenient choice for coupled-channel calculations. By selecting an initial $\left|F, m_{F}\right\rangle=|6,+6\rangle$ two-atom state with the two electronic and the two nuclear spins fully oriented, only $F=4,5$, or 6 bound levels should appear in the spectrum, the change in $F$ being at most 1 in each of the optical transitions. Together, the above choices for the initial and intermediate states lead to a considerable reduction of the complexity of the observed bound-state spectrum.

This paper is organized as follows. In Sec. II we describe our method of analysis, starting from IPA and including singlet-triplet mixing and accumulated phases. Section III describes the two-color photoassociation experiment. Section IV is devoted to the application of our method of analysis to 
this experiment. In Section V we formulate some conclusions.

\section{COUPLED SINGLET-TRIPLET METHOD OF ANALYSIS}

We introduce our method starting from the inverse perturbation analysis [1] and first taking into account coupled channels. The IPA is a method to improve a potential, in such a way that the corresponding Schrödinger equation reproduces as well as possible a set of experimental boundstate energies $E_{n}$ :

$$
\left[-\frac{\hbar^{2}}{2 \mu} \Delta+V(r)\right] \psi_{n}=E_{n} \psi_{n},
$$

with $\mu$ the reduced mass, equal to half the atomic mass in the present application. One makes a comparison with theoretical eigenvalues $E_{n}^{0}$ associated with an approximate potential $V^{0}(r)$ and its eigenfunctions $\psi_{n}^{0}$ :

$$
\left[-\frac{\hbar^{2}}{2 \mu} \Delta+V^{0}(r)\right] \psi_{n}^{0}=E_{n}^{0} \psi_{n}^{0} .
$$

Using first-order perturbation theory and an expansion of the difference potential $\Delta V=V-V^{0}$ in a set of suitable basis functions $g_{i}(r)[1,20]$, the energy differences are expressed as

$$
\Delta E_{n} \equiv E_{n}-E_{n}^{0}=\left\langle\psi_{n}^{0}|\Delta V| \psi_{n}^{0}\right\rangle=\sum_{i} c_{i}\left\langle\psi_{n}^{0}\left|g_{i}(r)\right| \psi_{n}^{0}\right\rangle .
$$

This set of linear equations for the unknown expansion coefficients $c_{i}$ is solved generally as a least-squares problem to construct a new theoretical potential $V^{0}(r)+\Delta V(r)$ and the whole procedure is restarted until one reaches convergence.

The IPA can only be applied to singlet and triplet potentials separately, since it assumes the absence of coupling. It therefore needs unmixed experimental singlet and triplet states as input. To introduce coupling, both Eqs. (1) and (2) are considered as coupled equations in the singlet-triplet or hyperfine basis, or any other basis. The IPA equation (3) can be carried over to the multichannel case essentially without change, $\psi_{n}^{0}$ now standing for a coupled state:

$$
\begin{aligned}
\Delta E_{n} & =\left\langle\psi_{n}^{0}|\Delta V| \psi_{n}^{0}\right\rangle \\
& =\sum_{i}\left[c_{i S}\left\langle\psi_{n}^{0}\left|P_{S} g_{i S}(r)\right| \psi_{n}^{0}\right\rangle+c_{i T}\left\langle\psi_{n}^{0}\left|P_{T} g_{i T}(r)\right| \psi_{n}^{0}\right\rangle\right],
\end{aligned}
$$

with $\Delta V$ an operator in spin space,

$$
\Delta V=P_{S} \Delta V_{S}(r)+P_{T} \Delta V_{T}(r),
$$

and $P_{S}$ and $P_{T}$ projection operators on the singlet and triplet spin subspaces, respectively.

The modification of the IPA to include coupling requires the tedious task of introducing a coupled-channel matrix structure into the previous equations and the associated com- puter code. This by itself produces a coupled generalization of the IPA, which to our knowledge has not been proposed or applied as an iterative method in the literature. In view of the cold-atom applications we have in mind, however, we are not interested in a coupled IPA, applicable to both the unmixed lower and mixed higher states. Instead we go on with a second modification. As pointed out in Sec. I a further ingredient of our approach is the replacement of the shortrange parts $r<r_{0}$ of the potentials $V_{S}(r)$ and $V_{T}(r)$ by a boundary condition at $r_{0}$, the largest interatomic distance where $S$ can still be considered a good quantum number due to the fact that the singlet-triplet energy splitting is large relative to the hyperfine constant $a_{h f}$ (see also Sec. I). The boundary condition takes the form of an accumulated phase $\phi_{S / T}$ of the corresponding rapidly oscillating radial wave functions $\psi_{S / T}(r)$ in each of the decoupled channels, defined by the WKB expression

$$
\psi\left(r_{0}\right)=A \frac{\sin \left[\int^{r_{0}} k(r) d r\right]}{k^{1 / 2}\left(r_{0}\right)} \equiv A \frac{\sin \phi\left(r_{0}\right)}{k^{1 / 2}\left(r_{0}\right)},
$$

$k(r)$ being the local radial wave number:

$$
k^{2}(r)=\frac{2 \mu}{\hbar^{2}}\left[E-V(r)-\frac{\hbar^{2} l(l+1)}{2 \mu r^{2}}\right] .
$$

In these equations we have omitted the subscript $S$ or $T$ for simplicity. This accumulated phase method has been introduced in Ref. [3,4]. Differentiating Eq. (6) we find that $\phi$ is related to the local logarithmic derivative by

$$
k \cot \phi=\frac{\psi^{\prime}}{\psi}+\frac{k^{\prime}}{2 k} .
$$

The validity of the WKB approximation is not a prerequisite for this approach: we could have specified the boundary condition by means of a logarithmic derivative of the radial wave function. We come back to this point later in this section. The accumulated phase may be considered as a convenient parametrization of the logarithmic derivative. Its convenience stems from its approximate linearity,

$$
\phi=\phi^{0}+E \phi^{E}+l(l+1) \phi^{l},
$$

over the relatively small $E$ and $l$ ranges near $E=l=0$, relevant for cold collisions, making it possible to fit experimental data with three parameters: $\phi^{0}, \phi^{E}, \phi^{l}$. In contrast, the logarithmic derivative shows the typical tangent-shaped excursions through infinity each time a radial node passes the point $r=r_{0}$. As an example we consider the ${ }^{85} \mathrm{Rb}$ case for which an analysis is presented here, based on accumulated phases at the radius $r_{0}=19 a_{0}$. Figure 2 shows $\phi_{S}$ and $\phi_{T}$ as a function of $E$ for $l=0$ (a) and as a function of $l(l+1)$ for $E=0$ (b), in both cases over ranges much larger than needed for the analysis in Sec. IV. The $E$ and $l(l+1)$ ranges covered by the actual measurement are indicated by the double-sided arrows in the graphs. Over this range the $E$ dependence is linear to within $\Delta \phi_{S}= \pm 6 \times 10^{-5}$ and $\Delta \phi_{T}= \pm 11 \times 10^{-5}$, 


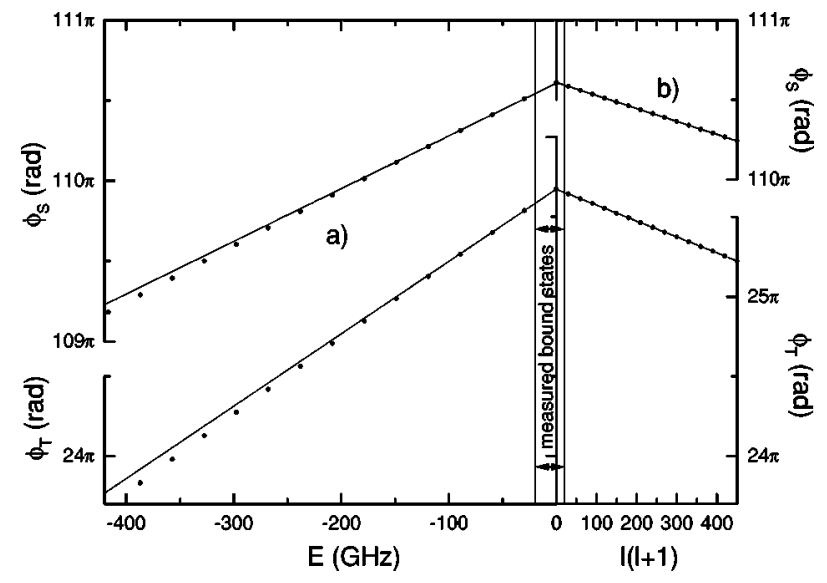

FIG. 2. (a) Accumulated phases $\phi_{S}(E, l=0)$ and $\phi_{T}(E, l=0)$ vs $E$ for IPA singlet and $a b$ initio triplet potentials. (b) Accumulated phases $\phi_{S}(E=0, l)$ and $\phi_{T}(E=0, l)$ vs $l(l+1)$ for the same potentials.

and the $l(l+1)$ dependence even to within $\Delta \phi_{S}= \pm 1$ $\times 10^{-6}$ and $\Delta \phi_{T}= \pm 3 \times 10^{-6}$. The graph for $\phi_{S}$ was calculated using Amiot's IPA singlet potential [21], that for $\phi_{T}$ using the Krauss and Stevens triplet potential [22]. In some of our previous analyses we also included higher-order derivative terms to extend the $E$ and $l$ ranges. Note that

$$
\tau=2 \hbar \frac{d \phi}{d E} \equiv 2 \hbar \phi^{E}
$$

is the classical time interval needed for the atoms to move from $r_{0}$ inward and back to $r_{0}$. Indeed, for the shallower triplet potential the phase is seen to be a steeper function of energy. Furthermore, the second derivative of $\phi_{S / T}(E)$ with respect to $E$ is seen to be negative, in agreement with the decreasing sojourn time left of $r_{0}$ for increasing energy.

A refinement that we introduce to increase the accuracy of our approach is to subtract not only $V(r)$ and the angular kinetic energy $\hbar^{2} l(l+1) / m r^{2}$ from the total energy $E$ to obtain the radial wave number (7) in the range $r<r_{0}$, but also a spin-energy term. The latter results from the total twoatom Zeeman interaction $V_{Z}$ and hyperfine interaction $V_{h f}$ $=\left(a_{h f} / \hbar^{2}\right)\left(\vec{s}_{1} \cdot \vec{i}_{1}+\vec{s}_{2} \cdot \vec{i}_{2}\right)$. At the interatomic distance $r_{0}$ the singlet-triplet energy separation is assumed to be so large that the singlet-triplet mixing, induced by the part $V_{h f}^{-}$ $=\frac{1}{2}\left(a_{h f} / \hbar^{2}\right)\left(\vec{s}_{1}-\vec{s}_{2}\right) \cdot\left(\vec{i}_{1}-\vec{i}_{2}\right)$ of $V_{h f}$ antisymmetric in the electronic spins, is negligible. Therefore the eigenvectors and eigenvalues of the sum $V_{h f}^{+}+V_{Z}$, diagonal in $S$, determine the spin states and spin energies of the decoupled channels. Here, $V_{h f}^{+}=\frac{1}{2} a_{h f} / \hbar^{2} \vec{S} \cdot \vec{I}$ is the remaining, symmetric, part of $V_{h f}$. The decoupled channels in the region $r<r_{0}$ will be denoted by the abbreviated notation $\alpha$ in the following ( $\alpha$ includes in particular the quantum number $S$ ).

The fact that we generally include a search for the above phase parameters in our approach implies that Eq. (4) has to be modified, since we want to derive a different set of parameters characterizing the modifications of the potentials from the discrepancies $\Delta E_{n}$ between theoretical and experi- mental eigenvalues. To include potential corrections in the interior region $r<r_{0}$ via changes of the accumulated phases, we have to find a solution for the following complications.

(1) The normalization of the state $\psi_{n}^{0}$ is tacitly assumed in the perturbation theory expression (4), although the phase description for the $r<r_{0}$ dynamics implies that the part of $\psi_{n}^{0}$ in the interior region is not explicitly dealt with. Rewriting Eq. (4) as

$$
\Delta E_{n}\left\langle\psi_{n}^{0} \mid \psi_{n}^{0}\right\rangle=\left\langle\psi_{n}^{0}|\Delta V| \psi_{n}^{0}\right\rangle,
$$

we need to deal with the part $\left\langle\psi_{n}^{0} \mid \psi_{n}^{0}\right\rangle_{r<r_{0}}$ of the normalization integral. This difficulty plays a role independent of whether a potential correction extends over $r<r_{0}$, over $r$ $>r_{0}$, or over both.

(2) $V_{S}(r)$ and $V_{T}(r)$ are continuous functions of $r$. When a potential correction in the exterior region extends up to $r_{0}$, this has consequences for the inner potentials, i.e., for the phase parameters.

Complication (1) is easily solved starting from the WKB expression (6) for the accumulated phase in one particular decoupled channel $\alpha$. Differentiating with respect to $E$ we have

$$
\begin{aligned}
\frac{\partial \phi_{\alpha}}{\partial E} & =\frac{2 \mu}{\hbar^{2}} \int^{r_{0}} \frac{1}{2 k_{\alpha}} d r \\
& \approx \frac{2 \mu}{\hbar^{2}} \int \frac{r_{0} \sin ^{2} \phi_{\alpha}(r)}{k_{\alpha}(r)} d r=\frac{2 \mu}{\hbar^{2} A_{\alpha}^{2}}\left\langle\psi_{n}^{0} \mid \psi_{n}^{0}\right\rangle_{\alpha, r<r_{0}} .
\end{aligned}
$$

The approximation involved in this equation is valid when $\sin ^{2} \phi_{\alpha}$ oscillates rapidly over a radial range where $k_{\alpha}$ is approximately constant, which corresponds to the condition of validity of the WKB approximation. Writing $\psi$ near $r_{0}$ in the form (6), we find $A_{\alpha}$. Equation (12) then allows us to express the part $\left\langle\psi_{n}^{0} \mid \psi_{n}^{0}\right\rangle_{r<r_{0}}$ of the normalization integral in terms of the derivatives $\partial \phi_{\alpha} / \partial E$. In connection with complication (2) we also need to express the part $\left\langle\psi_{n}^{0}|\Delta V| \psi_{n}^{0}\right\rangle_{r<r_{0}}$ in changes of the phase parameters. We use the WKB expression

$$
\begin{aligned}
\Delta \phi_{\alpha} & =-\frac{2 \mu}{\hbar^{2}} \int_{0}^{r} \frac{1}{2 k_{\alpha}} \Delta V_{\alpha} d r \\
& \approx-\frac{2 \mu}{\hbar^{2}} \int_{0}^{r \sin ^{2} \phi_{\alpha}(r)} k_{\alpha} \Delta V_{\alpha} d r \\
& =-\frac{2 \mu}{\hbar^{2} A_{\alpha}^{2}}\left\langle\psi_{n}^{0}\left|\Delta V_{\alpha}\right| \psi_{n}^{0}\right\rangle_{\alpha, r<r_{0}} .
\end{aligned}
$$

With these relations, the main equation of our method is found to be 


$$
\begin{aligned}
\Delta E_{n}\left(\left\langle\psi_{n}^{0} \mid \psi_{n}^{0}\right\rangle_{r>r_{0}}+\frac{\hbar^{2}}{2 \mu} \sum_{\alpha} A_{\alpha}^{2} \frac{\partial \phi_{\alpha}}{\partial E}\right) \\
=\sum_{i}\left[c_{i S}\left\langle\psi_{n}^{0}\left|P_{S} g_{i S}(r)\right| \psi_{n}^{0}\right\rangle_{r>r_{0}}\right. \\
\left.\quad+c_{i T}\left\langle\psi_{n}^{0}\left|P_{T} g_{i T}(r)\right| \psi_{n}^{0}\right\rangle_{r>r_{0}}\right]-\frac{\hbar^{2}}{2 \mu} \sum_{\alpha} A_{\alpha}^{2} \Delta \phi_{\alpha},
\end{aligned}
$$

where $\Delta \phi_{\alpha}$ is written in the form

$$
\Delta \phi_{\alpha}=\Delta \phi_{\alpha}^{0}+E_{n}^{0} \Delta \phi_{\alpha}^{E}+l(l+1) \Delta \phi_{\alpha}^{l},
$$

following from Eq. (9). Like the IPA equations (4) we started from, Eq. (14) is a set of linear equations for the unknown parameters (in this case $c_{i S}, c_{i T}, \Delta \phi_{\alpha}^{0}, \Delta \phi_{\alpha}^{E}$, and $\Delta \phi_{\alpha}^{l}$ ) in terms of the energy differences $\Delta E_{n}$. Again, we solve it as a least-squares problem. Clearly, in case some of the parameters are already known with sufficient accuracy from other sources, the corresponding terms in Eqs. (14) and (15) are replaced by 0 .

Note that Eq. (14) no longer contains the functions $g_{i s}(r)$ and $g_{i T}(r)$ in the interior region $r<r_{0}$, but only long-range dispersion and exchange terms represented by analytic expressions $g_{i S}(r), g_{i T}(r)$ for $r>r_{0}$, containing unknown dispersion and exchange parameters. This point is illustrated in more detail in Sec. IV. At this point we emphasize again that our method avoids the intricate instability and convergence problems of the IPA by replacing the nonunique "mathematical" basis functions $g_{i}(r)$ with accumulated phases and "physical" long-range interactions. Such problems played an important role in previous work of Moerdijk and coworkers, in which the IPA was applied to $\mathrm{Li}_{2}$ and $\mathrm{Na}_{2}$ bound states very close to the continuum [4]. Three further remarks are in place. The first relates to the channels $\alpha$. The label $\alpha$ distinguishes the various spin eigenstates of $V_{Z}+V_{h f}^{+}$, so that the dynamical problem is diagonal in $\alpha$ for $r<r_{0}$. For a vanishing or weak magnetic field, $\alpha$ corresponds to the combination of quantum numbers $S, I, F, m_{F}$. For a strong $B$ field each $\alpha$ contains a combination $S, I, m_{F}$, but a mixture of $F$ values.

The second remark relates to the use of the WKB approximation in the foregoing formulation. Our approach is most easily explained using the WKB approximation. We note, however, that the WKB approximation is not essential for the validity of the approach. To see this we start from an equation for the Wronskian of the unperturbed and perturbed states $\left|\psi_{n}^{0}\right\rangle$ and $\left|\psi_{n}\right\rangle$ :

$$
\begin{aligned}
& \frac{\partial}{\partial r} \sum_{\alpha}\left(\psi_{n \alpha}^{0} \frac{\partial}{\partial r} \psi_{n \alpha}-\psi_{n \alpha} \frac{\partial}{\partial r} \psi_{n \alpha}^{0}\right) \\
& \quad=\frac{2 \mu}{\hbar^{2}} \sum_{\alpha^{\prime} \alpha} \psi_{n \alpha^{\prime}}^{0}\left(\Delta V_{\alpha^{\prime} \alpha}-\Delta E_{n} \delta_{\alpha^{\prime} \alpha}\right) \psi_{n \alpha},
\end{aligned}
$$

following from the time-independent Schrödinger equations for $\left|\psi_{n}^{0}\right\rangle$ and $\left|\psi_{n}\right\rangle$. Here, the channel components $\psi_{n \alpha}(r)$ and $\psi_{n \alpha}^{0}(r)$ are chosen to be real. In Eq. (16) we have expanded $\left|\psi_{n}^{0}\right\rangle$ and $\left|\psi_{n}\right\rangle$ in the above orthonormal basis of spin states $|\alpha\rangle$ :

$$
\left|\psi_{n}^{0}\right\rangle=\sum_{\alpha} \psi_{n \alpha}^{0}(r)|\alpha\rangle, \quad\left|\psi_{n}\right\rangle=\sum_{\alpha} \psi_{n \alpha}(r)|\alpha\rangle
$$

According to Eqs. (4) and (5), the corresponding matrix elements $\Delta V_{\alpha^{\prime} \alpha}$ of $\Delta V$ are given by

$$
\Delta V_{\alpha^{\prime} \alpha}=\sum_{i}\left(c_{i S}\left\langle\alpha^{\prime}\left|P_{S}\right| \alpha\right\rangle g_{i S}+c_{i T}\left\langle\alpha^{\prime}\left|P_{T}\right| \alpha\right\rangle g_{i T}\right),
$$

with the functions $g_{i S}(r), g_{i T}(r)$ restricted to $r>r_{0}$.

Integrating over $r$ from $r_{0}$ to $\infty$, we find to first order in the modifications $\Delta V$ and $\Delta E_{n}$ :

$$
\begin{aligned}
\sum_{\alpha} & {\left[\psi_{n \alpha}^{0}\left(r_{0}\right)\right]^{2} \Delta \Lambda_{\alpha} } \\
& =\frac{2 \mu}{\hbar^{2}} \sum_{\alpha^{\prime} \alpha} \int_{r_{0}}^{\infty} \psi_{n \alpha^{\prime}}^{0}\left(\Delta V_{\alpha^{\prime} \alpha}-\Delta E_{n} \delta_{\alpha^{\prime} \alpha}\right) \psi_{n \alpha}^{0} d r,
\end{aligned}
$$

with $\Lambda_{\alpha}$ the logarithmic derivative of the radial wave function in channel $\alpha$ at $r_{0}$. This equation enables us to formulate the approach in terms of logarithmic derivatives. As pointed out above, however, the convenient properties of accumulated phases lead us to reformulate the approach by parametrizing the logarithmic derivative for each of the channels $\alpha$ via

$$
\Lambda=\frac{k\left(r_{0}\right)}{\tan \phi}-\frac{k^{\prime}\left(r_{0}\right)}{2 k\left(r_{0}\right)} .
$$

This definition of the accumulated phase has been used in all our previous work $[3,8-10,2]$. It corresponds to the integral $\int^{r_{0}} k(r) d r$ when the WKB approximation applies. Starting from Eq. (19), Eq. (14) with $A_{\alpha}^{2}$ replaced by $\left|\psi_{n \alpha}^{0}\left(r_{0}\right)\right|^{2} k_{\alpha} / \sin ^{2} \phi_{\alpha}$ can be derived by expressing the differentials $\Delta \Lambda_{\alpha}$ in the variations $\Delta \phi_{\alpha}$ and $\Delta E_{n}$ using Eq. (20). We emphasize that in this general form of a boundary condition the accumulated phase method is based only on the assumption of negligible singlet-triplet coupling in the range $r<r_{0}$. In practice, this condition is fulfilled by the choice of $r_{0}$ at an interatomic separation where the molecular singlet and triplet potential curves have an energy separation large compared to the strength of the hyperfine interaction.

As a third remark, we point out that the formalism presented above (and our computer code based on it) is flexible enough to contain a full coupled IPA as a special case. It can be realized by selecting an $r_{0}$ value close to the origin and choosing the differentials $\Delta \Lambda_{\alpha}$ to be zero. In Sec. IV, however, we describe the application of our method to the measured ${ }^{85} \mathrm{Rb}_{2}$ bound states near dissociation, making use of the formalism in the restricted accumulated phase form. 


\section{TWO-COLOR PHOTOASSOCIATION EXPERIMENT}

We have measured the energies of the highest bound states of ${ }^{85} \mathrm{Rb}_{2}$ with two-color photoassociation spectroscopy [2]. The experiment is very similar to our previous one-color photoassociation experiments [8-10,19]. About $10^{4}{ }^{85} \mathrm{Rb}$ atoms are transferred from a magneto-optic trap (MOT) to a far off-resonance optical dipole force trap (FORT) [23]. The atomic density is about $10^{12} \mathrm{~cm}^{-3}$ and the temperature is a few hundred microkelvin. The FORT is created from a 1.7 $\mathrm{W}$, linearly polarized laser detuned $35 \mathrm{~nm}$ to the red from the rubidium $\mathrm{D}_{2}$ line, focused to a $10 \mu \mathrm{m}$ Gaussian waist. This creates a trap with $12 \mathrm{mK}$ depth. The atoms are doubly spin polarized in the $f=3, m_{f}=3$ state by a repumper beam tuned to the ${ }^{85} \mathrm{Rb} 5^{2} S_{1 / 2}(f=2)$ to $5^{2} P_{3 / 2}(f=3)$ transition and an optical pumping beam tuned to the ${ }^{85} \mathrm{Rb} 5^{2} S_{1 / 2}(f=3)$ to $5^{2} P_{3 / 2}(f=3)$ transition. The optical pumping beam is circularly polarized with an intensity of $100 \mu \mathrm{W} / \mathrm{cm}^{2}$. A $3 \mathrm{G}$ magnetic field is applied parallel to the FORT and optical pumping beam propagation direction.

Once the FORT has been loaded, the FORT laser beam is alternated with two photoassociation laser beams and the optical pumping and repumper beams in $5 \mu$ s cycles for a total of $200 \mathrm{~ms}$. This is done to avoid the effect of the ac Stark shift of the trap laser on the photoassociation spectra and the optical pumping process. At the start of each cycle, only the FORT beam is applied for $2.5 \mu \mathrm{s}$. After this, the FORT beam is turned off, and only the optical pumping and repumper beams irradiate the atoms for $0.5 \mu \mathrm{s}$. For the last stage of each cycle, only the photoassociation beams are applied for $2.0 \mu \mathrm{s}$. Photoassociation laser beam 1, at frequency $\nu_{1}$, is ordinarily kept at a constant frequency that excites transitions to a $\left|0_{g}^{-}(v, J)\right\rangle$ state near the $5{ }^{2} S_{1 / 2}$ $+5^{2} P_{1 / 2}$ dissociation limit. Photoassociation laser beam 2, at frequency $\nu_{2}$, is tuned to the blue of $\nu_{1}$ (see Fig. 1 of Ref. [2]). Both photoassociation laser beams are collinear with the FORT beam, and are focused to a waist of $20 \mu \mathrm{m}$. Photoassociation beam 1 is supplied by a temperature and current tuned SDL-5401-G1 diode laser with an intensity of $1.6 \mathrm{~kW} / \mathrm{cm}^{2}$ and a linewidth of less than $20 \mathrm{MHz}$. Photoassociation beam 2 is supplied by a Ti:sapphire ring laser with an intensity of $30-200 \mathrm{~W} / \mathrm{cm}^{2}$ and linewidth less than 2 $\mathrm{MHz}$. After the $200 \mathrm{~ms}$ cycling process is complete, the number of atoms in the trap is measured with laser-induced fluorescence. This process is repeated for a succession of laser frequencies $\nu_{2}$.

As in the one-color experiments, the photoassociation beam 1 promotes trap loss when resonant with a free-bound transition. A pair of free atoms absorbs a photon from beam 1 to create a short-lived excited molecular state, which then usually spontaneously decays to a pair of free atoms with a kinetic energy high enough to escape from the trap. Figure 3 shows the one-color photoassociation spectrum obtained by scanning only one of the photoassociation beams across a single vibrational level at $12573.1 \mathrm{~cm}^{-1}$. The upward going peaks are associated with the trap loss induced by this laser. The $J=0,2$, and 4 rotational levels are visible in this spectrum. For the two-color spectra, photoassociation beam 1 is tuned to the maximum of the $J=2$ peak, and induces a con-

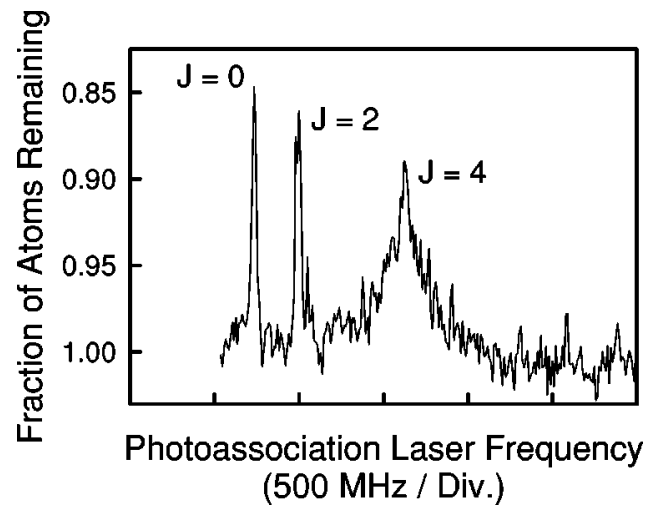

FIG. 3. One-color photoassociation spectrum of ${ }^{85} \mathrm{Rb}_{2}$. A single vibrational level near $12573.1 \mathrm{~cm}^{-1}$ is shown, with $J=0,2$, and 4 rotational levels visible.

stant loss of atoms (10-30\% depending on experimental conditions) in the absence of the second laser.

Figures 4(a) and 4(b) show the two-color photoassociation spectra with $\nu_{1}$ tuned to the $J=2$ rotational levels of two different vibrational levels at $12563.1 \mathrm{~cm}^{-1}$ and $12573.1 \mathrm{~cm}^{-1}$, respectively. As $\nu_{2}$ becomes resonant with a bound-bound transition between the excited state and a ground molecular state, the trap loss decreases. The positions of the ground-state vibrational levels are thus visible as downward going peaks in the two-color spectra. This occurs because the excited state is power broadened by photoassociation laser 2. This reduces the efficiency of excitation of the colliding atoms by photoassociation laser 1 , and therefore

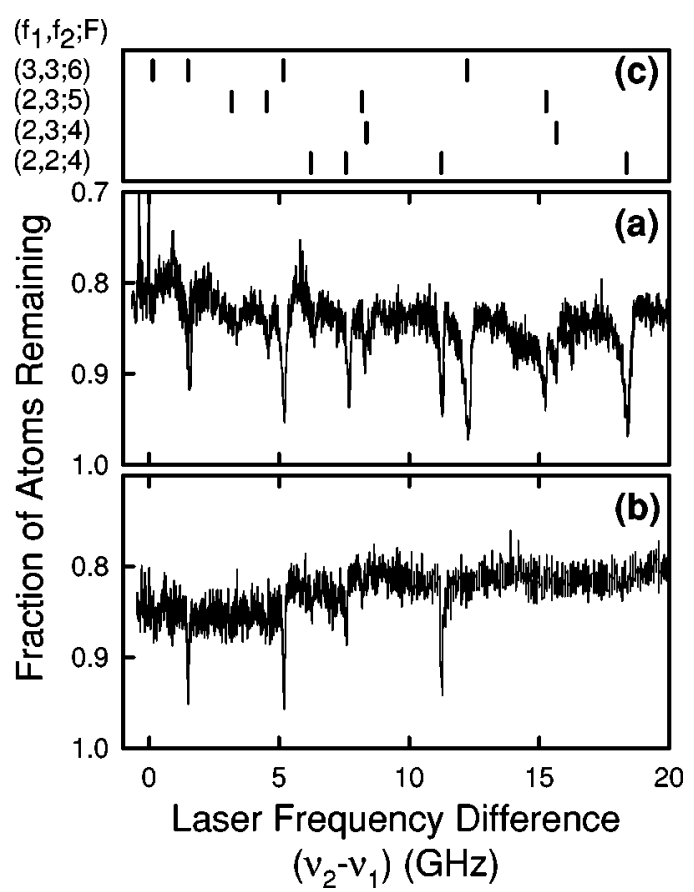

FIG. 4. Two-color photoassociation spectra with $\nu_{1}$ tuned to intermediate vibrational levels states near $12563.1 \mathrm{~cm}^{-1}$ (a) and $12573.1 \mathrm{~cm}^{-1}$ (b). The two-color spectrum is obtained by setting $\nu_{1}$ to the intermediate state $J=2$ rotational level, and scanning $\nu_{2}$ to the blue of $\nu_{1}$. (c) Assignments of the observed levels. 


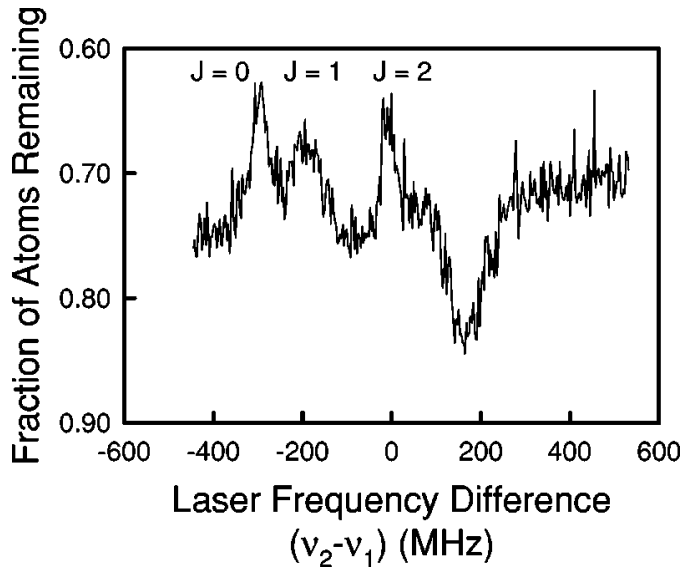

FIG. 5. High-resolution two-color photoassociation spectra for very small laser frequency difference $\left(\nu_{2}-\nu_{1}\right) . \nu_{1}$ is tuned to the $J=2$ intermediate state near $12563.1 \mathrm{~cm}^{-1}$. The data show three upward going peaks indicating further one-color trap loss as $\left|0_{g}^{-}(v, J=0,1,2)\right\rangle$ states are excited. The downward going peak shows the most weakly bound ground molecular state observed in this experiment, with a binding energy of $160 \mathrm{MHz}$.

reduces the trap loss. A theory of these two-color trap loss line shapes has been given by Bohn and Julienne [24]. Figure 5 shows a magnified view of the two-color photoassociation spectrum for very small positive and negative frequency differences $\nu_{1}-\nu_{2}$. For zero and negative frequency differences, upward going peaks are observed which are due to one-color trap loss induced by photoassociation laser 2 by transitions to $J=0,1$, and 2. (For this spectrum $J=1$ is visible because the atoms were not polarized.) For a positive frequency difference of about $160 \mathrm{MHz}$, the spectrum shows a downward going peak associated with the highest bound level observed in this experiment.

We investigated the first $20 \mathrm{GHz}$ below the $5{ }^{2} S_{1 / 2}(f$ $=3)+5^{2} S_{1 / 2}(f=3)$ dissociation limit. The intermediate state at $12563.1 \mathrm{~cm}^{-1}$ resulted in a spectrum showing more ground molecular states than the state at $12573.1 \mathrm{~cm}^{-1}$. We also observed a few of these same levels with an intermediate state at $12561.8 \mathrm{~cm}^{-1}$. From these photoassociation spectra, the binding energies of 12 ground-state levels were measured, as shown in Table I. We searched for but did not find molecular states with binding energies greater than 20 $\mathrm{GHz}$, presumably due to small Franck-Condon factors.

The frequency scan of photoassociation laser 2 was calibrated to an accuracy of $\pm 20 \mathrm{MHz}$ with a scanning Michelson interferometer wavemeter and a $300 \mathrm{MHz}$ free spectral range étalon. The zero of the frequency difference $\nu_{1}-\nu_{2}$ was determined by the point at which the second laser induced one-color trap loss on the $0_{g}^{-}(J=2)$ line, as in Fig. 5. Photoassociation laser 1 was passively stabilized and demonstrated drift below $20 \mathrm{MHz}$ over the course of a scan.

The widths of the observed lines varied from about 60 $\mathrm{MHz}$ to about $300 \mathrm{MHz}$. The widths of the broadest lines were probably dominated by power broadening, whereas for the narrowest lines the thermal width of the initial continuum state plays a significant role. To our knowledge there are no explicit calculations for thermally averaged two-color photoassociation line shapes in the literature. However, one-color thermally averaged photoassociation line shapes have been calculated previously $[8-10,25]$. These calculations show that the photoassociation peaks can easily be shifted by $(1-2) k_{B} T$ relative to the peak position in the absence of thermal broadening effects, where $T$ is the temperature of the gas and $k_{B}$ is Boltzmann's constant. For our conditions this shift would be in the range from about 6 to $20 \mathrm{MHz}$. Similar

TABLE I. Spectrum of ${ }^{85} \mathrm{Rb}_{2} \quad l=2, F=4,5,6$ levels observed experimentally, including assignments of $\left(f_{1}, f_{2}\right)$ progressions and integer parts of the $v$ quantum number relative to the dissociation limit $v_{D}$ for the progression involved. Theoretically predicted levels without $\left(E_{t h, 1}\right)$ and with $\left(E_{t h, 2}\right)$ Feshbach resonance data taken into account. The $l=4, F=6 g$-wave shape resonance state observed by one-color spectroscopy is also included.

\begin{tabular}{ccccccc}
\hline \hline$F$ & $l$ & $E_{\text {exp }}(\mathrm{GHz})$ & $E_{t h, 1}(\mathrm{GHz})$ & $E_{t h, 2}(\mathrm{GHz})$ & $v-v_{D}$ & $\left(f_{1}, f_{2}\right)$ \\
\hline 6 & 4 & $+0.015 \pm 0.002$ & +0.014 & +0.015 & -1 & $(3,3)$ \\
6 & 2 & $-0.16 \pm 0.06$ & -0.14 & -0.14 & -1 & $(3,3)$ \\
5 & 2 & & -3.18 & -3.18 & -1 & $(2,3)$ \\
4 & 2 & $-6.23 \pm 0.06$ & -6.22 & -6.22 & -1 & $(2,2)$ \\
6 & 2 & $-1.52 \pm 0.06$ & -1.48 & -1.46 & -2 & $(3,3)$ \\
5 & 2 & $-4.58 \pm 0.06$ & -4.51 & -4.50 & -2 & $(2,3)$ \\
4 & 2 & $-7.61 \pm 0.06$ & -7.56 & -7.54 & -2 & $(2,2)$ \\
6 & 2 & $-5.20 \pm 0.06$ & -5.13 & -5.09 & -3 & $(3,3)$ \\
5 & 2 & & -8.17 & -8.12 & -3 & $(2,3)$ \\
4 & 2 & $-8.34 \pm 0.06$ & -8.35 & -8.33 & -3 & $(2,3)$ \\
4 & 2 & $-11.27 \pm 0.06$ & -11.23 & -11.18 & -3 & $(2,2)$ \\
6 & 2 & $-12.22 \pm 0.06$ & -12.24 & -12.14 & -4 & $(3,3)$ \\
4 & 2 & & -12.50 & -12.43 & -4 & $(3,3)$ \\
5 & 2 & $-15.24 \pm 0.06$ & -15.28 & -15.18 & -4 & $(2,3)$ \\
4 & 2 & $-15.67 \pm 0.06$ & -15.67 & -15.61 & -4 & $(2,3)$ \\
4 & 2 & $-18.39 \pm 0.06$ & -18.38 & -18.29 & -4 & $(2,2)$ \\
\hline \hline
\end{tabular}


shifts should occur for our two-color spectra, and they should occur in both the calibration spectrum (as in Fig. 5), which determines the zero of the laser difference frequency, as well as in the observed lines. In addition, line pulling due to drifts in the frequency of laser 1 and ac Stark shifts could also play a role in the position of these lines. These shifts are difficult to evaluate accurately since the tuning of laser 1 and the gas temperature are not accurately known, and since we did not attempt to model the line shapes. However, they could easily be on the order of the width of the lines. Due to these uncertainties, we consider our earlier statement [2] of error bounds of $\pm 30 \mathrm{MHz}$ for some of the lines to be insufficiently conservative, and have chosen to apply a common error estimate of $\pm 602 \phi \mathrm{MHz}$ for all levels in the reanalysis of our data presented below.

\section{APPLICATION OF COUPLED ANALYSIS TO TWO-COLOR PHOTOASSOCIATION EXPERIMENT}

We assign the quantum numbers of the observed bound states as follows. As pointed out earlier, due to the abovementioned selection rule $l=J$, which is valid also in the downward transition, we produce $l=2$ bound ground-state $\mathrm{Rb}_{2}$ levels only, since a $J=2$ rotational level of the lower $0_{g}^{-}$ electronic state is excited as an intermediate state. Also, with a two-photon transition from the initial $\left|F, m_{F}\right\rangle=|6,+6\rangle$ state of two doubly polarized atoms, only $F=4,5$, or 6 levels can be formed with $m_{F} \geqslant 4$. Note furthermore that for all $B, f_{1}$ and $f_{2}$ are good quantum numbers if $F=5$ or 6 . In addition, for $l$ even Bose symmetry excludes $F=5$ for $\left(f_{1}, f_{2}\right)=(3,3)$. We should therefore expect to see two mutually shifted pure triplet vibrational progressions converging to the $\left(f_{1}, f_{2}\right)=(3,3)$ hyperfine threshold for $F=6$ and to the $(2,3)$ hyperfine threshold for $F=5$, respectively. These are the two sequences indicated as $(3,3 ; 6)$ and $(2,3 ; 5)$ in Fig. 4(c). The energy differences between pairs of corresponding levels in the progressions indeed correspond to the singleatom hyperfine splitting, $3.04 \mathrm{GHz}$. The remaining levels must be assigned $F=4$ and have mixed singlet-triplet character. In view of this one would expect these levels to display a less regular pattern than the $F=5$ and 6 levels. Anticipating our further analysis we note, however, that $f_{1}$ and $f_{2}$ continue to be approximately good quantum numbers [26] for the mixed singlet-triplet states because of an approximate equality of singlet and triplet phases for ${ }^{85} \mathrm{Rb}$ (see also Tsai et al. [2]). This equality is similar to that which has been discovered for ${ }^{87} \mathrm{Rb}$ [27-29], and in fact follows from it by mass scaling of the wave function phases. We note that this scaling of small phase differences is not expected in general, but just happens to occur for the particular case of $\mathrm{Rb}$. As a consequence, the $F=4$ states display a pattern almost as regular as the $F=5$ and 6 states. Table I shows the spectrum of $l=2, F=4,5,6$ levels observed in our experiment, including the assignments of the $\left(f_{1}, f_{2}\right)$ progressions and the integer parts of the differences $v-v_{D}$, with $v$ the vibrational quantum number and $v_{D}$ its generally nonintegral value at the dissociation limit for the progression involved. We also include the $l=4, F=6 \quad g$-wave shape resonance state observed by one-color spectroscopy [10], which will also be used in the following analysis.

We analyze the energy spectrum as follows. Referring to Sec. II, we characterize the singlet potential in the interval $0<r<19 a_{0}$ by the singlet accumulated phase $\phi_{S}^{0}$, which we take as a fit parameter, and by its derivatives $\phi_{S}^{E}$ and $\phi_{S}^{l}$, calculated on the basis of the above-mentioned IPA potential of Amiot [21]. In the same interval the triplet interaction is characterized by three similar parameters: $\phi_{T}^{0}$ is taken as a fit parameter, whereas $\phi_{T}^{E}$ and $\phi_{T}^{l}$ are taken from the Krauss and Stevens $a b$ initio triplet potential [22]. For $r>19 a_{0}$ we write the interaction operator as

$$
V(r)=-\frac{C_{6}}{r^{6}}-\frac{C_{8}}{r^{8}}-\frac{C_{10}}{r^{10}}+V_{e x c h}+V_{h f} .
$$

For $C_{6}$ we consider values in a range that includes at the lower end the interval 4550 100 a.u., determined in a previous one-color photoassociation experiment [10], and at the higher end the interval $4700 \pm 50$ a.u. from the analysis in Ref. [30]. Values for $C_{8}$ and $C_{10}$ are taken from Marinescu et al. [31]. The exchange interaction is taken from Hadinger and Hadinger [32].

The actual application of our coupled analysis consists of a number of iterations, in each of which Eq. (19) is solved as a least-squares problem. At the beginning of an iteration step, eigenfunctions $\psi_{i}^{0}$ and eigenvalues $E_{i}^{0}(i=1, \ldots, M)$ are calculated for certain phase and potential parameters $x_{j}(j=1, \ldots, N, N<M)$. This gives us a vector $\underline{\Delta E}$ having the set of differences with the experimental energies $\Delta E_{i}$ as components, from which the vector $\underline{\Delta x}$ with the changes $\Delta x_{j}$ as components is to be determined. Writing Eq. (19) in the form

$$
\underline{\Delta E}=\underline{\underline{M}} \underline{\Delta x}
$$

we use the same wave functions $\psi_{i}^{0}$ to calculate the $M \times N$ matrix $M$. To take into account the experimental error bars we divide the components $\Delta E_{i}$ and $M_{i j}$ by the $i$ th error bar without changing our notation. The least-squares solution is found by solving Eq. (22) for the parameter changes $\underline{\Delta x}$ using the pseudoinverse of $\underline{\underline{M}}$ (see Ref. [33]):

$$
\underline{\Delta x}=\underline{\underline{M}}^{-1} \underline{\Delta E} .
$$

This iteration step is repeated until convergence has been reached. It turns out that highly accurate values of the elements of $\underline{M}$ are needed for correct and rapid convergence. This is especially true for directions in parameter space for which the least-squares sum varies slowly. We have paid special attention to this aspect by studying the $M=N=1$ case. In our coupled analysis the $g$-wave shape resonance is included as a quasibound state, by assuming its wave function to vanish at a radius far inside the centrifugal barrier.

Parenthetically, we note that our method is easily extended to cases where (part of) the measured data consist of energy differences between states without information on absolute positions with respect to the dissociation threshold, for instance. In such cases the differences between elements of 
TABLE II. Iteration paths for three choices of starting points in parameter space $\left(C_{6}, \phi_{S}^{0}, \phi_{T}^{0}\right)$, given in the first line. The values in the second to fourth lines are obtained by the coupled singlet-triplet analysis. Accumulated phases $\phi_{S}^{0}$ and $\phi_{T}^{0}$ for zero energy and angular momentum are given modulo $\pi$. The dispersion coefficient $C_{6}$ is given in atomic units.

\begin{tabular}{ccccccccc}
\hline \hline$C_{6}$ & $\phi_{S}^{0}$ & $\phi_{T}^{0}$ & $C_{6}$ & $\phi_{S}^{0}$ & $\phi_{T}^{0}$ & $C_{6}$ & $\phi_{S}^{0}$ & $\phi_{T}^{0}$ \\
\hline 4700 & 2.42739 & 2.69209 & 4600 & 2.86411 & 3.08447 & 4600 & 3.07411 & 3.08447 \\
4652 & 2.64742 & 2.88692 & 4650 & 2.65240 & 2.89221 & 4647 & 2.66936 & 2.90512 \\
4651 & 2.65024 & 2.89024 & 4651 & 2.65031 & 2.89033 & 4651 & 2.65035 & 2.89038 \\
4651 & 2.65017 & 2.89019 & 4651 & 2.65024 & 2.89026 & 4651 & 2.65036 & 2.89037 \\
\hline \hline
\end{tabular}

$\Delta E$ and between elements of $M$ are used in Eqs. (22) and (23) instead of the elements themselves.

Since we have normalized the components of $\underline{\Delta E}$ to the measured energy uncertainty intervals, the covariance matrix for the parameter values finally obtained is given by

$$
\frac{\chi^{2}}{M-N}\left(\underline{\underline{M}}^{T} \underline{M}^{-1}\right.
$$

the diagonal elements of which give the square of the final parameter errors. In this expression $\chi^{2}$ is the least-squares sum.

In view of the large discrepancy between the results of our previous paper [2] and the later results obtained by Roberts et al. [30], we here repeat the analysis of Ref. [2] but now without a restriction on $C_{6}$. Also, as noted above, we keep $\phi_{T}^{E}$ fixed to the $a b$ initio value. We thus treat $C_{6}, \phi_{S}^{0}$, and $\phi_{T}^{0}$ as parameters in a fit to the two-photon data in combination with the energy position of the $g$-wave shape resonance. Only one $g$ function in Eqs. (14) and (18) is therefore involved in the analysis: $g_{i S}(r)=g_{i T}(r)=1 / r^{6}$ and $c_{i S}=c_{i T}$ $=-C_{6}$. We emphasize again that this choice is very different from the usual IPA functions. While the triplet parameters are primarily determined by the $F=5$ and 6 levels including the g-wave shape resonance, the singlet phase parameter $\phi_{S}^{0}$ is mostly determined by the mixed singlettriplet $F=4$ states, in particular those with $\left(f_{1}, f_{2}\right)=(2,3)$. The starting point of an iteration was chosen in the $C_{6}$ range between 4450 and 4750 a.u. mentioned above and in the $\phi_{S}^{0}, \phi_{T}^{0}$ ranges suggested by analyses of previous cold-atom experiments [8,9,27-29] involving ${ }^{85} \mathrm{Rb}$ and ${ }^{87} \mathrm{Rb}$, as well as by direct inspection of the level energies, as we mentioned in the beginning of this section. The iteration is ended when the steps in the phase values and $C_{6}$ become an order of magnitude smaller than the uncertainties in these parameters following from the experimental error bars. This generally occurred within three steps. As an illustration, Table II shows the iteration paths in parameter space for three starting points. The phase values are given modulo $\pi$. The end points of these iterations coincide within the above maximum final step values and represent an absolute $\chi^{2}$ minimum. As usual in $\chi^{2}$ searches, not every choice for the starting point leads to this minimum. Sometimes convergence takes place to a local minimum with a considerably higher $\chi^{2}$. This is generally due to one or more levels that are misidentified between theory and experiment. It results in sets of optimal parameters and error bars completely different from those at the absolute $\chi^{2}$ minimum.

We find $C_{6}=4650 \pm 50$ a.u., a range with a considerable overlap with the interval $4700 \pm 50$ a.u. from Ref. [30]. Considering the $C_{6}$ range $4550 \pm 100$ a.u. found by Boesten et al. [10], which is based on independent information, i.e., a Franck-Condon oscillation in the photoassociation spectrum, one would conclude that our central value $C_{6}=4650$ a.u. may be considered as the optimal overall result, if the Franck-Condon oscillation is taken into account. The theoretical level energies that follow from this analysis are given in the fourth column $\left(E_{t h, 1}\right)$ of Table I. For varying $C_{6}$ near the above central value $\bar{C}_{6}=4650$ a.u. we subsequently derive optimal values of $\phi_{S}^{0}$ and $\phi_{T}^{0}$. The corresponding vibrational quantum numbers at dissociation $v_{D S}(\bmod 1)$ and $v_{D T}(\bmod 1)$ are

$$
\begin{aligned}
& v_{D S}=+0.0050+\left(1.8 \times 10^{-4}\right)\left(C_{6}-\bar{C}_{6}\right) \pm 0.0095, \\
& v_{D T}=-0.0518+\left(1.0 \times 10^{-4}\right)\left(C_{6}-\bar{C}_{6}\right) \pm 0.0032,
\end{aligned}
$$

with the $C_{6}$ values in a.u.

The analysis of Ref. [30] included the parameters of a magnetic-field-induced Feshbach resonance that has been observed in the ${ }^{85} \mathrm{Rb}+{ }^{85} \mathrm{Rb}$ elastic scattering channel by two groups $[11,30]$ since the experimental two-color photoassociation work that we analyzed above. As a following step therefore we supplement the foregoing analysis by including the extremely accurate values of the resonance field $B_{\text {peak }}$ $=155.2 \pm 0.4 \mathrm{G}$ and the resonance "width" $\Delta=11.6$ $\pm 0.5 \mathrm{G}$, measured by Roberts et al. [30] as additional experimental data in our parameter search with corresponding coupled-channel $\underline{M}$ matrix elements. We thus find a $\chi^{2}$ minimum at $C_{6}=47 \overline{00} \pm 50$ a.u., in agreement with Ref. [30] and consistent with the above $C_{6}=4650$ a.u. value. The set of theoretical energy levels obtained in the overall fit are included in Table I (fifth column: $E_{t h, 2}$ ).

We also give the optimal $v_{D S}, v_{D T}$ values for varying $C_{6}$ near the central value $\bar{C}_{6}=4700$ a.u. of the latter fit:

$$
\begin{aligned}
& v_{D S}=+0.0090+\left(0.8 \times 10^{-4}\right)\left(C_{6}-\bar{C}_{6}\right) \pm 0.0009, \\
& v_{D T}=-0.0568+\left(1.4 \times 10^{-4}\right)\left(C_{6}-\bar{C}_{6}\right) \pm 0.0011 .
\end{aligned}
$$

The corresponding scattering lengths are (in a.u.) 


$$
\begin{gathered}
a_{S}=+2650-12.93\left(C_{6}-\bar{C}_{6}\right) \pm 250, \\
a_{T}=-361-1.17\left(C_{6}-\bar{C}_{6}\right) \pm 10 .
\end{gathered}
$$

The agreement between the experimental and theoretical energies is quite good. Relative to the theoretical energies $E_{t h, 1}$, the measured level energies show a small systematic difference of $-20 \mathrm{MHz}$, and a random scatter of about $\pm 30 \mathrm{MHz}$, within our measurement error. For the set of theoretical energies $E_{t h, 2}$, the systematic difference increases to $-63 \mathrm{MHz}$, and the scatter remains about $\pm 30 \mathrm{MHz}$. The systematic difference is probably due to line shape effects, as discussed in the experimental Sec. III. For completeness we point out that the above analysis following Eq. (24), including only the bound-state data, yields the values $B_{\text {peak }}$ $=148 \pm 10 \mathrm{G}, \Delta=9 \pm 4 \mathrm{G}$.

The analysis of the two-color photoassociation experiment presented in this section illustrates how our coupled analysis is applied in practice. We believe that the method will be useful also for further work on the extraction of interactions between cold atoms from highly excited bound diatomic states.

Parenthetically, we note that the sensitivity of $v_{D S}$ and $v_{D T}$ to $C_{6}$, described by the second terms on the right-hand side of Eqs. (25) and (26), can be determined either by completing the coupled iteration successively for various choices of $C_{6}$ or, more easily, by making use of our coupled approach. The formalism presented in Sec. II allows us to consider $C_{6}$ or other parameters formally as parameters $x_{j}$ and to translate their variations $\underline{\Delta x}$ into variations $\underline{\Delta E}$ by means of the corresponding $M$ matrix elements calculated in our coupled approach. The latter in turn can be translated into variations of $v_{D S}$ and $v_{D T}$ or any other parameters determined in the coupled search, using the inverse equation (23). This procedure is applicable generally in the case of parameters for which one wants to indicate the dependence of the final results explicitly as in Eqs. (25) and (26) instead of including them in the parameter search.

\section{CONCLUSIONS}

We have described a coupled singlet-triplet analysis for extracting information on interactions between cold atoms from energies of bound diatomic states. Like the IPA it is based on inverse perturbation theory, but unlike the IPA it is applicable in situations where external and internal (electronic and nuclear spin) degrees of freedom of the two bound atoms are coupled. Basically, the purpose of the approach is to extrapolate interaction properties from just below the dissociation threshold to just above, i.e., the cold-collision regime. Although the method is applied here to bound states of two identical ground-state alkali-metal atoms, it is applicable to any pair of cold atoms where the short-range interactions are taken into account in terms of a boundary condition on the radial wave functions at a certain interatomic distance $r_{0}$. In our case of two alkali-metal atoms the boundary condition takes the form of an accumulated phase for the wave function in the singlet and triplet spin channels. The latter description derives its usefulness from the fact that cold collisions together with the considered highest part of the boundstate spectrum comprise an energy range small compared to the typical relative kinetic energies of the two atoms in the distance range $r<r_{0}$. We have made clear that the validity of the WKB approximation for $r<r_{0}$ is not essential.

We illustrated the coupled analysis by an application to the bound ${ }^{85} \mathrm{Rb}_{2}$ states measured in a two-color photoassociation experiment and a $g$-wave shape resonance observed in a one-color photoassociation experiment. We also performed a combined parameter search by including the measured resonance field and resonance width of a recently observed Feshbach resonance.

\section{ACKNOWLEDGMENTS}

We gratefully acknowledge the support of the work at Texas by the R. A. Welch Foundation, the U.S. National Science Foundation, and the NASA Microgravity Research Division. The work at Eindhoven is part of the research program of the Stichting FOM, which is financially supported by NWO.
[1] W. M. Kosman and J. Hinze, J. Mol. Spectrosc. 56, 93 (1975); C. R. Vidal and H. Scheingraber, ibid. 65, 46 (1977).

[2] C. C. Tsai, R. S. Freeland, J. M. Vogels, H. M. J. M. Boesten, B. J. Verhaar, and D. J. Heinzen, Phys. Rev. Lett. 79, 1245 (1997).

[3] B. J. Verhaar, K. Gibble, and S. Chu, Phys. Rev. A 48, R3429 (1993).

[4] A. J. Moerdijk, W. C. Stwalley, R. G. Hulet, and B. J. Verhaar, Phys. Rev. Lett. 72, 40 (1994); A. J. Moerdijk, B. J. Verhaar, and A. Axelsson, Phys. Rev. A 51, 4852 (1995).

[5] L. Li, A. M. Lyyra, W. T. Luh, and W. C. Stwalley, J. Chem. Phys. 93, 8452 (1990); G. Zhao, W. T. Zemke, J. T. Kim, B. Ji, H. Wang, J. T. Bahns, W. C. Stwalley, L. Li, A. M. Lyyra, and C. Amiot, ibid. 105, 7976 (1996).

[6] P. D. Lett, P. S. Julienne, and W. D. Phillips, Annu. Rev. Phys. Chem. 46, 423 (1995).

[7] D. J. Heinzen, in Atomic Physics 14, edited by D. J. Wineland,
C. E. Wieman, and S. J. Smith AIP Conf. Proc. No. 323 (AIP, New York, 1995), pp. 369-388; D. J. Heinzen, Int. J. Mod. Phys. B 11, 3297 (1997).

[8] J. R. Gardner, R. A. Cline, J. D. Miller, D. J. Heinzen, H. M. J. M. Boesten, and B. J. Verhaar, Phys. Rev. Lett. 74, 3764 (1995).

[9] H. M. J. M. Boesten, C. C. Tsai, B. J. Verhaar, and D. J. Heinzen, Phys. Rev. Lett. 77, 5194 (1996).

[10] H. M. J. M. Boesten, C. C. Tsai, J. R. Gardner, D. J. Heinzen, and B. J. Verhaar, Phys. Rev. A 55, 636 (1997).

[11] Ph. Courteille, R. S. Freeland, D. J. Heinzen, F. A. van Abeelen, and B. J. Verhaar, Phys. Rev. Lett. 81, 69 (1998).

[12] H. M. J. M. Boesten, C. C. Tsai, D. J. Heinzen, A. J. Moonen, and B. J. Verhaar, J. Phys. B 32, 287 (1999).

[13] E. Tiesinga, C. J. Williams, P. S. Julienne, K. M. Jones, P. D. Lett, and W. D. Phillips, J. Res. Natl. Inst. Stand. Technol. 101, 505 (1996). 
[14] E. R. I. Abraham, W. I. McAlexander, J. M. Gerton, R. G. Hulet, R. Côté, and A. Dalgarno, Phys. Rev. A 53, R3713 (1996).

[15] K. M. Jones, S. Maleki, L. P. Ratliff, and P. D. Lett, J. Phys. B 30, 289 (1997).

[16] E. R. I. Abraham, W. I. McAlexander, C. A. Sackett, and R. G. Hulet, Phys. Rev. Lett. 74, 1315 (1995); E. R. I. Abraham, W. I. McAlexander, J. M. Gerton, R. G. Hulet, R. Côté, and A. Dalgarno, Phys. Rev. A 55, R3299 (1997).

[17] M. Movre and G. Pichler, J. Phys. B 10, 2631 (1977).

[18] C. J. Williams and P. S. Julienne, J. Chem. Phys. 101, 2634 (1994).

[19] R. A. Cline, J. D. Miller, and D. J. Heinzen, Phys. Rev. Lett. 73, 632 (1994); 73, 2636(E) (1994).

[20] Note that the choice of basis functions generally used in applications of IPA is different from that in our approach to be described below.

[21] C. Amiot, J. Chem. Phys. 93, 8591 (1990).

[22] M. Krauss and W. J. Stevens, J. Chem. Phys. 93, 4236 (1990).

[23] J. D. Miller, R. A. Cline, and D. J. Heinzen, Phys. Rev. A 47,
R4567 (1993).

[24] J. L. Bohn and P. S. Julienne, Phys. Rev. A 54, R4637 (1996).

[25] R. Napolitano, J. Weiner, C. J. Williams, and P. S. Julienne, Phys. Rev. Lett. 73, 1352 (1994).

[26] J. M. Vogels, B. J. Verhaar, and R. H. Blok, Phys. Rev. A 57, 4049 (1998).

[27] S. J. J. M. F. Kokkelmans, H. M. J. M. Boesten, and B. J. Verhaar, Phys. Rev. A 55, R1589 (1997).

[28] P. S. Julienne, F. H. Mies, E. Tiesinga, and C. J. Williams, Phys. Rev. Lett. 78, 1880 (1997).

[29] J. P. Burke, Jr., J. L. Bohn, B. D. Esry, and C. H. Greene, Phys. Rev. A 55, R2511 (1997).

[30] J. L. Roberts, N. R. Claussen, J. P. Burke, Jr., C. H. Greene, E. A. Cornell, and C. E. Wieman, Phys. Rev. Lett. 81, 5109 (1998).

[31] M. Marinescu, H. R. Sadeghpour, and A. Dalgarno, Phys. Rev. A 49, 982 (1994).

[32] G. Hadinger and G. Hadinger, J. Mol. Spectrosc. 175, 441 (1996)

[33] R. Penrose, Proc. Cambridge Philos. Soc. 51, 406 (1955). 